\title{
Blood Lactate Level as Predictor of Early Outcome after Cardiac Surgery under Cardiopulmonary Bypass
}

\author{
MD. ABUL KALAM AZAD, MD. ABUL QUASHEM, MD. REZAUL KARIM, MD. KAMRUL HASAN \\ Department of Cardiac Surgery, National Institute of Cardiovascular Disease (NICVD), Sher-e-bangla Nagar, Dhaka. \\ Address of Correspondence: Md. Kamrul Hasan, Professor \& Head, Dept. of Cardiac Surgery, NICVD, Dhaka, \\ E-mail:profkamrulcts@gmail.com
}

\begin{abstract}
:
We examined the hypothesis that high blood lactate level in ICU patient after adult cardiac surgery under cardiopulmonary bypass is associated with early adverse outcome. The objective of this study was to evaluate whether blood lactate level after cardiac surgery is predictor of the early outcome after adult cardiac surgery under CРB. In this prospective observational study total 100 patients were enrolled who underwent elective cardiac surgery under CPB as per inclusion and exclusion criteria. Blood lactate levels $>3$ mmol/ L 6 hours after ICU transfer were present in 57(57\%) patients. The binary logistic regression analysis showed that blood lactate level 6 hours after ICU transfer is an independent predictor for prolonged mechanical ventilation time (OR 2.417, 95\% CI 1.272 - 4.596, $p=.007)$, prolonged ICU stay (OR 1.562, 95\% CI 1.181 - 2.067, $p=.002)$, neurological deficit (OR 2.432, 95\% CI 1.539 - 3.843, $p=.001)$, pulmonary complication (OR 1.301, 95\% CI 1.011 - 1.676, p =.041), arrhythmia (OR 1.444, 95\% CI 1.102 - 1.893, p $=.008)$, renal dysfunction (OR 1.838, 95\% CI $1.352-2.497, p=.001)$ and mortality (OR 1.822, 95\% CI 1.123- 2.955, $p=.015)$. In conclusion, blood lactate level 6 hours after ICU transfer is an independent risk factor for worse outcomes in adult patients including mortality after cardiac surgery under CPB.
\end{abstract}

Key Words: Blood Lactate Level, Cardio-Pulmonary ByPass.

University Heart Journal 2017; 13(2): 50-54

\section{Introduction:}

High blood lactate level is a well-recognized marker of circulatory failure, and its severity has been associated with morbidity and mortality in different clinical conditions. ${ }^{1,2}$ After cardiac surgery, hyperlactatemia (HL) is relatively common ${ }^{3}$ and is associated with morbidity and mortality. 4

Irrespective of its origin, hyperlactataemia and its persistence have been demonstrated to be an early indicator of worse outcome in cardiac surgical patients. ${ }^{5}$ The liver removes $70 \%$ of lactate. Within the Periportal hepatocytes, metabolism involves the processes of gluconeogenesis. The liver receives $25 \%$ of cardiac output. Only when the liver blood flow is reduced to $25 \%$ of normal is there a reduction in lactate clearance. Under anaerobic conditions, glycolysis becomes the predominant mode of hepatic energy production. As such, the liver becomes a lactate-producing organ rather than using lactate for gluconeogenesis. ${ }^{6}$

Various preoperative factors or comorbidities may create the right environment for $\mathrm{HL}$ during $\mathrm{CPB}$. Age, female gender, congestive heart failure, low left ventricular ejection fraction, hypertension, atherosclerosis, diabetes, preoperative low hemoglobin value, redo or complex surgery, and emergency procedures were found to be risk factors for HL.7 Cardiologic arrest induces anaerobic myocardial metabolism with a net production of lactate from glycolysis. Persistent lactate release during reperfusion suggests a delayed recovery of normal aerobic metabolism and may lead to depressed myocardial function. ${ }^{8}$

In current practice, lactate is frequently measured usually with the goal of detecting tissue hypoxia during ICU care. However, high blood lactate levels in ICU are getting more importance to predict early outcome after cardiac surgery in recent publications. The aim of this study was to evaluate whether blood lactate level after cardiac surgery is predictor of early adverse outcomes.

\section{Methods:}

This prospective observational study was carried out in the department of Cardiac Surgery at NICVD, Sher-ebangla Nagor Dhaka from July, 2013 to June 2014. 


\section{Inclusion criteria:}

1. All the adult patients irrespective of sex undergoing elective CPB during cardiac surgery.

\section{Exclusion criteria:}

1. Patients with pre-CPB high blood lactate level

2. Emergency surgery

3. Redo surgery

4. Adult complex congenital heart defects

5. Hepatic dysfunction

6. End-stage renal disease

7. Intraoperative mortality or mortality less than 6 hours after ICU transfer.

8. Patient's refusal for enrollment in the study.

Considering the inclusion and exclusion criteria, a total number of 100 consecutive adult patients age ranged from 18 to 70 years of both sexes undergoing elective cardiac surgery under cardiopulmonary bypass were included. Demographic data like age, sex, BMI were recorded. Risk factors profile including diabetes mellitus, hypertension, dyslipidemia and impaired LVEF were noted. Patients were grouped into group A (Lactate $<3 \mathrm{mmol} / \mathrm{L}$ ): Those patients who had less than $3 \mathrm{mmol} / \mathrm{L}$ of blood lactate level 6 hours after transfer in ICU and group B (Lactate $>3 \mathrm{mmol} / \mathrm{L}$ ): Those patients who had $>3 \mathrm{mmol} / \mathrm{L}$ of blood lactate level 6 hours after transfer in ICU.

\section{Operative Procedure}

\section{Anesthesia}

All patients undergoing cardiac surgery were given general anesthesia with intubation under standard anesthetic techniques.

Cardiopulmonary bypass

All patients were treated with the same CPB technique. Mean systemic arterial pressure was continuously monitored and maintained between 60 and $80 \mathrm{~mm} \mathrm{Hg}$. During perfusion, HCT was maintained around (25-28\%).

\section{Measurement of Blood Lactates}

Arterial blood samples were analyzed by blood gas analyzer, model - Siemens RAPIDlab 1265, manufactured by Beckman int. California, USA.

\section{ICU Management}

Standard ICU management protocols were used and the patients were subsequently shifted to Post-ICU and then to ward or cabin and discharged whenever appropriate according to operating consultant's judgment.

\section{Follow up}

All patients were closely followed up and Data were recorded using a pre-formed case record form (questionnaire) up to the time of discharge from hospital, and at the time of 1st Follow up after 1 month of surgery.

\section{Statistical analysis}

Data was processed using SPSS (Statistical Package for Social Science) version 17.0. Potential risk factors were assessed using a $x^{2}$ test, Fisher's exact test, and t test. A forward multiple logistic regression analysis was then performed to estimate independent predictive factors for complications. For all analysis a $p$-value $<0.05$ were considered statistically significant.

\section{Results:}

A total of 100 patients were enrolled in this study from which $57 \%$ patients were in high lactate group. Death occurred in $7(7 \%)$ patients. There was no significant difference in demographic variables and co-morbidities (Table -I).

The patients in group $\mathrm{B}$ had a longer duration of operation time, $\mathrm{CPB}$ time and ACC time. The Operation time was $319.02 \pm 70.479$ in group $A$ and $359.47 \pm 83.274$ in group $\mathrm{B}(p=.012), \mathrm{CPB}$ time was $121.65 \pm 43.449$ in group $\mathrm{A}$ and $141.88 \pm 42.312$ in group B $(p=.021)$ and ACC time was $72.88 \pm 29.124$ in group A and $86.32 \pm 26.156$ in group $\mathrm{B}(p=.017)$ (Table -II).

Regarding the outcome variables in this study, we found in group A and group B neurological deficits [1(2.3\%) vs $12(21.1 \%) ; \mathrm{p}=0.006]$, pulmonary complication [9(20.9\%) vs $17(29.8 \%) ; p=0.001]$, arrhythmia [4(9.3\%) vs $19(33.3 \%) ; \mathrm{p}=0.005]$, renal dysfunction [ $5(11.6 \%)$ vs $23(40.4 \%) ; \mathrm{p}=0.002$ ] (Table - III)

In binary logistic regression analysis shows that blood lactate level 6 hours after ICU transfer is an independent predictor for prolonged mechanical ventilation time (OR $2.417,95 \%$ CI $1.272-4.596, \mathrm{p}=.007)$, prolonged ICU stay (OR 1.562, 95\% CI $1.181-2.067, \mathrm{p}=.002$ ), neurological deficit (OR 2.432, 95\% CI 1.539 - 3.843, $\mathrm{p}=.001)$, pulmonary complication (OR 1.301, 95\% CI 1.011 -1.676, p - .041), arrhythmia (OR 1.444, 95\% CI 1.102 - 1.893, p - .008), renal dysfunction (OR 1.838, $95 \%$ CI $1.352-2.497, \mathrm{p}=.001)$ and mortality (OR 1.822, $95 \%$ CI 1.123- 2.955, $\mathrm{p}=.015)$ (Table - IV) 
Table-I

Preoperative variables screening findings

\begin{tabular}{|c|c|c|c|c|c|}
\hline \multirow[t]{2}{*}{ Variables } & & \multicolumn{2}{|c|}{ Lactate Group (mmol/L) } & \multirow[b]{2}{*}{ Total } & \multirow[b]{2}{*}{ P Value } \\
\hline & & $\begin{array}{c}\text { Group A } \\
(n=43)<3\end{array}$ & $\begin{array}{c}\text { Group B } \\
(n=57) \geq 3\end{array}$ & & \\
\hline Age\# & $>60$ years & $4(9.3 \%)$ & $5(8.8 \%)$ & $9(9 \%)$ & $1.000^{\mathrm{NS}}$ \\
\hline Sex* & Female & $18(41.9 \%)$ & $20(35.1 \%)$ & $38(38 \%)$ & $0.490^{\mathrm{NS}}$ \\
\hline BMI* & Obese \&Overweight & $4(9.3 \%)$ & $9(15.8 \%)$ & $13(13 \%)$ & $.340^{\mathrm{NS}}$ \\
\hline $\mathrm{DM} *$ & & $11(25.6 \%)$ & $14(24.6 \%)$ & $25(25 \%)$ & $.907^{\mathrm{NS}}$ \\
\hline HTN* & & $7(16.3 \%)$ & $7(12.3 \%)$ & $14(14 \%)$ & $.568^{\mathrm{NS}}$ \\
\hline Dyslipidemia* & & $8(18.6 \%)$ & $9(15.8 \%)$ & $17(17 \%)$ & $.711^{\mathrm{NS}}$ \\
\hline ImpairedLVEF\# & & $5(11.6 \%)$ & $6(10.5 \%)$ & $11(11 \%)$ & $1.000^{\mathrm{NS}}$ \\
\hline
\end{tabular}

Data were analyzed using *Chi-Square test, \#Fisher Exact test: Level of significance was 0.05. $(\mathrm{n}=$ number of patients, NS= not significant), BMI, body mass index; DM, Diabetes mellitus; HTN, Hypertension; LVEF, Left ventricular ejection fraction.

\section{Table-II}

Comparison of Operation time, CPB time, ACC time between groups

\begin{tabular}{|c|c|c|c|}
\hline \multirow{3}{*}{ Peroperative Variables } & \multicolumn{2}{|c|}{ Lactate Group (mmol/L) } & \multirow{3}{*}{$P$ Value } \\
\hline & Group A $(n-43)<3$ & Group B $(n=57)>3$ & \\
\hline & Mean \pm SD & Mean \pm SD & \\
\hline Operation time* & $319.02 \pm 70.479$ & $359.47 \pm 83.274$ & $.012^{s}$ \\
\hline CPB time* & $121.65 \pm 43.44972 .88$ & $141.88 \pm 42.312$ & $.021^{s}$ \\
\hline ACC time* & \pm 29.124 & $86.32 \pm 26.156$ & $.017^{s}$ \\
\hline
\end{tabular}

\# Data were analyzed using't' test, ( $\mathrm{n}=$ number of patients, $\mathrm{S}=$ significant), CPB, Cardiopulmonary bypass; ACC, Aortic cross clamp.

Table-III

Postoperative variables

\begin{tabular}{|c|c|c|c|c|}
\hline \multirow[t]{2}{*}{ Variables } & \multicolumn{2}{|c|}{ Lactate Group (mmol/L) } & \multirow[b]{2}{*}{ Total } & \multirow[b]{2}{*}{$P$ Value } \\
\hline & Group $\mathrm{A}(\mathrm{n}=43)<3$ & Group $B(n=57)>3$ & & \\
\hline LOS & $08(18.6 \%)$ & $36(63.1 \%)$ & $44(44 \%)$ & $<.0001 *$ \\
\hline Prolonged MVT & $0(0 \%)$ & $6(10.5 \%)$ & $6(6 \%)$ & $.036^{s}$ \\
\hline Prolonged ICU stay & $7(16.3 \%)$ & $18(31.6 \%)$ & $25(25 \%)$ & $.080^{\mathrm{TM}}$ \\
\hline Reoperation for bleeding & $0(0 \%)$ & $3(5.3 \%)$ & $3(3 \%)$ & $.257^{N S}$ \\
\hline Neurological deficits & $1(2.3 \%)$ & $12(21.1 \%)$ & $13(13 \%)$ & $.006^{s}$ \\
\hline Pulmonary complication & $9(20.9 \%)$ & $17(29.8 \%)$ & $26(26 \%)$ & $.001^{s}$ \\
\hline Arrhythmia & $4(9.3 \%)$ & $19(33.3 \%)$ & $23(23 \%)$ & $.005^{s}$ \\
\hline Renal dysfunction & $5(11.6 \%)$ & $23(40.4 \%)$ & $28(28 \%)$ & $.002^{s}$ \\
\hline Mortality & $1(2.3 \%)$ & $6(10.5 \%)$ & $7(7 \%)$ & $.234^{N S}$ \\
\hline
\end{tabular}

Data were analyzed using *Chi-Square test, \#Fisher Exact test; Level of significance was 0.05. ( $\mathrm{n}=$ number of patients, NS = not significant); LOS, Low output syndrome; MVT, Mechanical ventilation time; ICU, Intensive care unit. 
Table-IV

Binary Logistic Regression Analyses of Predictors for adverse outcome

\begin{tabular}{llccccc}
\hline Input variable & Outcome Variables & $\mathrm{B}$ & $P$ value & OR & $95.0 \%$ Lower & C.I. Upper \\
\hline Blood & LOS & .247 & .108 & 1.281 & .947 & 1.732 \\
lactate 6h & Prolong MVT & .883 & .007 & 2.417 & 1.271 & 4.596 \\
after ICU & Prolong ICU stay & .446 & .002 & 1.562 & 1.181 & 2.067 \\
transfer & Reoperation for bleeding & .385 & .260 & 1.469 & .753 & 2.869 \\
& Neurological deficit & .889 & .001 & 2.432 & 1.539 & 3.843 \\
& Pulmonary complication & .263 & .041 & 1.301 & 1.011 & 1.676 \\
& Arrhythmia & .368 & .008 & 1.444 & 1.102 & 1.893 \\
& Renal complication & .608 & .001 & 1.838 & 1.352 & 2.497 \\
& Mortality & .600 & .015 & 1.822 & 1.123 & 2.955 \\
\hline
\end{tabular}

LOS, Low output syndrome; MVT, Mechanical ventilation time; ICU, Intensive care unit; OR, Odds ratio; CI, Confidence interval.

\section{Discussion:}

This study has demonstrated that a lactate level $>3 \mathrm{mmol} /$ $\mathrm{L}$ at 6 hours after ICU transfer are related to postoperative adverse events and is independently associated with risk of major complication after cardiac surgery.

Outcome after cardiac operation is determined by preoperative characteristics of the patients in addition to intra operative factors such as operation time, CPB time, and ACC time. The mean with standard deviation of these characteristics were recorded. The difference between the two groups is statistically significant which indicates that operation time; $\mathrm{CPB}$ time and $\mathrm{ACC}$ time are one of the important causes of hyperlactatemia. Similarly, Rao et al (2001), Hajjar et al (2013), Maillet et al (2003) and Ranucci et al (2006) stated that there is significant correlation between CPB time and postoperative adverse outcome..$^{4,5,8,9}$ Maillet et al (2003) and Demers et al (2000) also found ACC time as an important peroperative factor that had statistically significant difference between two groups. In current study, operation time is found as a risk factors causing postoperative high lactate and is statistically significant. 4,7

The present study also showed that the difference between two groups is statistically significant in prolong mechanical ventilation. Maillet et al (2003) showed in previous study that prolong mechanical ventilation and ICU stay found in hyperlactatemia group after cardiac surgery. ${ }^{4}$

In this study in case of low output syndrome and arrhythmia the difference between the two groups are statistically significant. Rao et al (2001) and Arnold et al (2009) stated that the development of postoperative LOS and cardiovascular complications in the absence of an intraoperative misadventure revealed a failure of myocardial protection causing persistent anaerobic metabolism and lactate release. ${ }^{8,10}$

In our study we evaluate the outcome variables for major adverse outcome and found neurological deficit, pulmonary complication and renal dysfunction have statistically significant differences between groups, Nichol et al (2010) showed a strong positive correlation between blood lactate levels and the risk of morbidity and mortality with neurological deficits.11 Hajjar et al (2013), Malliet et al (2003) and Ranucci et al (2006) showed in their studies a strong correlation between high lactate and major complications after cardiac surgery that are similar with current study. $4,5,9$

In our study, binary logistic regression analysis shows that blood lactate level 6 hours after ICU transfer is an independent predictor for prolonged mechanical ventilation time, prolonged ICU stay, neurological deficit, pulmonary complication, arrhythmia, renal dysfunction and mortality. The results of this study and discussion thereof prompt us to recommend that routine lactate level measurement is essential for postoperative cardiac ICU to detect early adverse outcome.

\section{Limitations of the study}

The main limitation of the study is the absence of randomization. The study has been prospectively designed; but no real randomization has been performed. Finally, it is performed in a single center, which could restrict the generalization of our findings.

\section{Conclusion:}

The findings of this study permit to conclude that blood lactate level 6 hours after ICU transfer is associated with an increased risk of postoperative morbidity and 
mortality after cardiac surgery. It reveals that after balancing the cofounding variables, blood lactate level after ICU transfer will be remain as predictor of adverse outcome. So, normolactatemia after cardiac surgery is essential to achieve the aim to maintain tissue perfusion and to minimize postoperative morbidity and mortality.

\section{References:}

1. Weil, M.H. and Afifi, A.A. Experimental and clinical studies on lactate and pyruvate as indicators of the severity of acute circulatory failure (shock), Circulation 1970;41:989-1001.

2. Rashkin, M.C., Bosken, C. and Baughman, R.P. Oxygen delivery in critically ill patients. Relationship to blood lactate and survival. Chest, 1985;87:580-84.

3. Takala, J., Uusaro, A., Parviainen, I. and Ruokonen, E. Lactate metabolism and regional lactate exchange after cardiac surgery, New Horiz, 1996;4:483-92.

4. Maillet, J-M., Le Besnerais, P. and Cantoni, M. Frequency, risk factors, and outcome of hyperlactatemia after cardiac surgery, Chest, 2003;123:1361-66.

5. Ranucci, M., De Toffol, B. and Isgro, G. Hyperlactatemia during cardiopulmonary bypass: determinants and impact on postoperative out-come, Critical Care, 2006;10:167.
6. Phypers, B. and Pierce, J.T. Lactate physiology in health and disease, the British Journal of Anaesthesia, Crtical Care and Pain, 2006;06(3):128-32.

7. Demers, P., Elkouri, S., Martineau, R., Couturier, A. and Cartier, R. Outcome with high blood lactate levels during cardiopulmonary bypass in adult cardiac surgery, The Annals of Thoracic Surgery, 2000;70:2082-86.

8. Rao, V., Ivanov, J., Weisel, R.D., Cohen, G., Borger, M.A. and Micule, D.A.G. Lactate release during reperfusion predicts low cardiac output syndrome after coronary bypass surgery, The Annals of Thoracic Surgery, 2001;71(6):1925-30.

9. Hajjar, L.A., Almeida, J.P., Fukushima, J.T., Rhodes, A., Vincent, J.L., Osawa, E.A. and Galas, F.R.B.G. High lactate levels are predictors of major complications after cardiac surgery, The Journal of Thoracic and Cardiovascular Surgery, 2013;146:455-60.

10. Arnold, R.C., Shapiro, N.I., Jones, A.E., Schorr, C., Pope, J., Casner, E., Parrillo, J.E., Dellinger, R.P. and Trzeciak, S. Multicenter study of early lactate clearance as a determinant of survival in patients with presumed sepsis, Shock, 2009;32(1);35-39.

11. Nichol, A.D., Egi, M., Pettila, V., Bellomo, R., French, C., Hart, G., Davies, A., Stachowski, E., Reade, M.C., Bailey, M. and Cooper, DJ. Relative hyperlactatemia and hospital mortality in critically ill patients: a retrospective multi-centre study, Critical Care, 2010; 14: R25. 